\title{
Elastic fair rate allocation scheme for multi-radio wireless mesh networks
}

\begin{abstract}
Fair bandwidth distribution among users remains a persistence issue in Wireless Mesh Networks (WMNs). Users which are more hops away from the gateway generally suffer from higher throughput degradation and hence higher delay. This is mainly due to the nature of IEEE 802.11 protocol, i.e. its operation in contention mode. In this paper, we propose a practical network-layer solution to address this fairness issue in Multi-Radio WMNs. The proposed framework consists of two main components: 1) lightweight messaging system and 2) Elastic fair Rate Allocation Scheme for Mesh Stations (ERASMeS). A highlight of the proposed solution is that it does not require any alteration of existing MAC and routing mechanisms and can be applied to nodes with multiple radio interfaces. This capability distinguishes it from other similar work on fairness provisioning in WMNs. The results obtained from our test-bed show that the proposed solution achieves a high degree of improvement in terms of end-to-end throughput and fairness index for all users.
\end{abstract}

Keyword: WMN; Multi-radio; Fairness; Throughput 